\title{
Avaliação das características físico-químicas e fitoquímicas de diferentes tinturas de barbatimão (Stryphnodendron barbatiman)
}

\author{
Patrícia da Fonseca*, Ana Paula Landi Librandi \\ Departamento de Física e Química, Faculdade de Ciências Farmacêuticas de Ribeirão Preto, \\ Universidade de São Paulo
}

*Correspondência:

P. Fonseca

Departamento de Física e Química

Faculdade de Ciências Farmacêuticas

de Ribeirão Preto

Universidade de São Paulo

Av. do Café, s/no - Monte Alegre

14040-903 - Ribeirão Preto - SP,

Brasil

E-mail: pafonseca@fcfrp.usp.br
Como conseqüencia da grande difusão e utilização de plantas medicinais, as indústrias vêm fabricando produtos a base de espécies vegetais, de diversas formas farmacêuticas, que têm sido comercializados em farmácias e casas de produtos naturais. No entanto, não há garantia para a grande maioria destes produtos, quanto à sua eficácia, segurança e qualidade, podendo trazer riscos à saúde do consumidor. Assim, torna-se importante o estabelecimento de protocolos padronizados de controle de qualidade para produtos fitoterápicos. Encontram-se disponiveis no mercado brasileiro tinturas de barbatimão provenientes de diversos fabricantes. Com a finalidade de avaliar a diferença entre a qualidade destas tinturas, foi realizado um estudo comparativo das características físico-químicas e fitoquímicas de tinturas de barbatimão provenientes de quatro fabricantes. Para a avaliação físico-química foram realizados ensaios de $\mathrm{pH}$, densidade, resíduo seco e doseamento de taninos. A análise fitoquímica foi feita empregando cromatografia em camada delgada. As diferenças físico-químicas e fitoquímicas observadas evidenciaram a falta de padronização na produção destas tinturas.
Unitermos

- Barbatimão

- Tintura/análise físicoquímica

\section{INTRODUÇÃO}

O consumo de plantas medicinais in natura ou devidamente preparadas vem apresentando um crescimento considerável em diversos países. Essa tendência pode ser explicada por diferentes fatores, destacando-se entre eles o custo elevado e os efeitos indesejáveis dos fármacos sintéticos, preferência dos consumidores por "produtos naturais", a certificação científica das propriedades farmacológicas de espécies vegetais, o desenvolvimento de novos métodos analíticos colocados à disposição do Controle de Qualidade, o desenvolvimento de novas formas de preparação e adminis- tração de produtos fitoterápicos, um melhor conhecimento químico, farmacológico e clínico das drogas vegetais e seus derivados (Canigueral et al., 2003; Di Stasi, 1996; Vieira, 2001). No entanto, a expansão deste setor farmacêutico não vem sendo acompanhada da adequada produção de fitoterápicos, no que se refere aos critérios recomendados de eficácia, segurança e qualidade (Newall; Anderson; Phillipson, 1996), estabelecidos pela Resolução da Diretoria Colegiada - $\mathrm{RDC} \mathrm{n}^{\circ}$ 48, da Agência Nacional de Vigilância Sanitária (ANVISA, 2004).

A avaliação da qualidade de uma tintura inicia-se com a análise da matéria prima, atentando-se principalmente para 
a identificação botânica ou zoológica (Desmichelle, 1987). Após o processo de produção, as tinturas devem passar por vários ensaios dentre eles: identificação, características organolépticas, densidade, resíduo seco, determinação do teor alcoólico e doseamento de compostos marcadores, como taninos, flavonóides, entre outros. Todos estes ensaios são importantes para assegurar o padrão de qualidade, atendendo a uma especificação pré-estabelecida (Prista, Alves, Morgado, 1979). No Brasil, devido à falta de ação de órgãos governamentais para controle da identidade e padronização de marcas e produtos, verifica-se que tinturas provenientes de diferentes fornecedores apresentam alta variabilidade (Pozetti, Cabrera, Bernardi, 1986).

Stryphnodendron barbatiman, popularmente conhecida como barbatimão, é uma planta medicinal rica em taninos que cresce no cerrado brasileiro, desde o Pará na região Amazônica até o Planalto Central alcançando o Sudeste (Minas Gerais e São Paulo) (Felfili et al., 1999). Esta espécie tem como sinonímias científicas Stryphnodendron adstringens (Mart.) Coville, Mimosa Vell. barbadetiman Vell e Stryphnodendron ovobatum Menth. (Lorenzi, 1992). As atividades farmacológicas do barbatimão estão diretamente relacionadas aos teores de taninos condensados. $\mathrm{O}$ decocto e o infuso preparados a partir da casca têm sido utilizados na medicina popular para o tratamento de distúrbios gastrointestinais, cicatrização de feridas, como antiinflamatório, antimicrobiano e antioxidante (Guarim Neto, 1987; Lopes et al., 2005; Panizza et al., 1988). No entanto, são relatadas variações no conteúdo destas substâncias (10-37\%) presentes na casca, dependendo do local de coleta do material (Teixeira, Soares, Scolforo, 1990). Um estudo comparativo da composição de taninos de três espécies de barbatimão (Stryphnodendron adstringens, Stryphnodendron polyphyllum e Dimorphandra mollis) foi realizado empregando-se ensaios colorimétricos e métodos cromatográficos (cromatografia em camada delgada e em papel), revelando diferenças significativas na composição química entre os gêneros Stryphnodendron e Dimorphandra (Santos et al., 2002). Além disto, a análise de frações de taninos revelou a presença de vários flavonóides e proantocianidinas com unidades pirogalol biologicamente ativas (De Mello, Petereit, Nahrsedt, 1996). Em decorrência desses altos teores, o barbatimão também tem sido empregado na indústria de couro e fabricação de tintas, demonstrando sua importância não só no campo da fitoterapia, mas também como fonte de taninos para abastecimento de curtumes e matéria-prima para indústrias de tintas (Rizzini, Mors, 1976).

Taninos são substâncias fenólicas solúveis em água com massa molecular entre 300 e 3000 Dalton, que apresentam a habilidade de formar complexos insolúveis em água com alcalóides, gelatina e outras proteínas. Uma técnica colorimétrica amplamente empregada para a quantificação de taninos é a reação com reagente de Folin-Ciocalteau, a qual se baseia no princípio de que em meio alcalino os fenóis reduzem a mistura dos ácidos fosfotúngstico e fosfomolíbdico (Monteiro et al., 2006). O Folin-Denis é uma metodologia bastante semelhante ao Folin-Ciolcateau, mas com a desvantagem da formação de um precipitado que interfere nas leituras espectrofotométricas (Singleton, Orthofer, LamuelaRaventós, 1999). Outro método descrito em alguns trabalhos emprega o Azul da Prússia, o qual tem como inconveniente o elevado tempo de reação (Magalhães, Rodrigues, Durães, 1997). Além disto uma combinação de ensaios colorimétricos e técnicas cromatográficas (Cromatografia em Camada Delgada ou Cromatografia em Papel) fornecem informações suficientes para distinguir entre as diferentes espécies de barbatimão, conforme resultados obtidos por Santos e colaboradores (2002).

Neste contexto, o objetivo deste estudo foi avaliar as possíveis diferenças nos parâmetros qualitativos e quantitativos de amostras de tintura de barbatimão disponíveis no mercado, enfocando principalmente as características físico-químicas e fitoquímicas, uma vez que não existem relatos na literatura de estudo similar envolvendo a tintura de barbatimão.

\section{MATERIAL E MÉTODOS}

\section{Obtenção das tinturas}

Amostras de tinturas de barbatimão provenientes de quatro fabricantes foram obtidas, nas suas embalagens originais, em farmácias de manipulação da cidade de Ribeirão Preto, São Paulo, Brasil e identificadas como A, B, C e D.

\section{Análise físico-química}

A análise físico-química das tinturas foi realizada determinando-se densidade, resíduo seco e pH através de técnicas preconizadas pela Farmacopéia Brasileira (1988) e o teor de taninos foi determinado conforme estabelecido pela European Pharmacopoeia (2005). Todos os ensaios foram realizados em duplicata e os resultados obtidos foram analisados comparando-se os valores obtidos para média e desvio padrão relativo (DPR).

\section{Análise de densidade}

Foram empregados balões volumétricos de $10 \mathrm{~mL}$ devidamente limpos e secos. Foram realizadas as pesagens dos balões volumétricos vazios e preenchidos com tintura de barbatimão empregando balança de precisão de $0,0001 \mathrm{~g}$. 

fórmula:

Para cálculo da densidade empregou-se a seguinte

Densidade $(\mathrm{g} / \mathrm{mL})=\frac{\mathrm{M} 1-\mathrm{M}}{10 \mathrm{~mL}}$

Onde:

M 1 - massa do balão contendo a tintura de barbatimão

$\mathrm{M}$ - massa do balão vazio

\section{Determinação de resíduo seco}

Para determinação do resíduo seco foi empregado aparelho de infravermelho (Gehaka, modelo IV2000) na seguinte configuração: módulo sólidos, temperatura de 120 ${ }^{0} \mathrm{C}$ na função auto dryer, sendo o resíduo seco expresso em porcentagem $(\%)$.

\section{Determinação do $\mathrm{pH}$}

Para a determinação do $\mathrm{pH}$ foi empregado $\mathrm{pHmetro}$ (Marconi, modelo MAPA200), sendo utilizados $20 \mathrm{~mL}$ de cada tintura de barbatimão.

\section{Determinação de taninos}

A determinação de taninos foi realizada empregando o reagente Folin-Ciocalteau. Preparou-se uma solução contendo aproximadamente $3 \mathrm{~g}$ de tintura de barbatimão em balão de $250 \mathrm{~mL}$, empregando água como diluente. Esta solução foi filtrada em papel de $125 \mathrm{~mm}$, empregando funil e coletada em erlenmeyer de $250 \mathrm{~mL}$, descartando-se os primeiros $50 \mathrm{~mL}$ (filtrado 1).

Para análise de polifenóis totais:

Diluíram-se $5 \mathrm{~mL}$ do filtrado 1 em balão volumétrico de $25 \mathrm{~mL}$, empregando água como diluente (solução 1). Transferiram-se $2 \mathrm{~mL}$ da solução 1 para balão volumétrico de $25 \mathrm{~mL}$, adicionou-se $1 \mathrm{~mL}$ do reagente de Folin Ciocalteau e $10 \mathrm{~mL}$ de água. Em seguida, completou-se o volume com solução de carbonato de sódio 290 g/L. Após 30 min de repouso, efetuou-se a leitura da absorbância (760 $\mathrm{nm}$ ) em espectrofotômetro UV-Vis (Shimadzu, modelo 1650 PC).

Para análise de polifenóis não adsorvidos em pó de pele:

Em $10 \mathrm{~mL}$ do filtrado 1 adicionou-se $0,1 \mathrm{~g}$ de pó de pele, agitou-se vigorosamente em agitador magnético por 60 min. Após este tempo, filtrou-se e obteve-se o filtrado 2. Diluíram-se $5 \mathrm{~mL}$ do filtrado 2 em balão volumétrico de 25 $\mathrm{mL}$, empregando água como diluente (solução 2). Transferiram-se $2 \mathrm{~mL}$ da solução 2 para balão volumétrico de 25 $\mathrm{mL}$, adicionou-se $1 \mathrm{~mL}$ do reagente de Folin-Ciocalteau e
$10 \mathrm{~mL}$ de água. Em seguida, o volume foi completado com solução de carbonato de sódio $290 \mathrm{~g} / \mathrm{L}$ e após $30 \mathrm{~min}$ efetuou-se a leitura da absorbância (760 nm).

Preparo da solução padrão:

Foi preparada imediatamente antes da leitura solução de ácido pirogálico (aproximadamente $50 \mathrm{mg} / 100 \mathrm{~mL}$, empregando água como diluente), denominada solução 3. Diluíram-se $5 \mathrm{~mL}$ da solução 3 em balão volumétrico de $100 \mathrm{~mL}$, empregando água como diluente (solução 4). Transferiram-se $2 \mathrm{~mL}$ da solução 4 para balão volumétrico de $25 \mathrm{~mL}$, adicionou-se $1 \mathrm{~mL}$ do reagente de Folin Ciocalteau e $10 \mathrm{~mL}$ de água, posteriormente completou-se o volume com solução de carbonato de sódio 290 g/L. Após 30 min efetuou-se a leitura da absorbância $(760 \mathrm{~nm})$.

Para determinação de taninos foi empregada a seguinte fórmula:

Teor de taninos $(\%)=\frac{62,5(\mathrm{~A} 1-\mathrm{A} 2) \mathrm{M} 2}{\mathrm{~A} 3 \times \mathrm{M} 1}$

Onde:

A1 - Absorbância de polifenóis totais

A2 - Absorbância de polifenóis não adsorvidos em pó de pele

A3 - Absorbância do ácido pirogálico

M1 - Tomada de ensaio da tintura de barbatimão

M2 - Massa de ácido pirogálico

\section{Análise fitoquímica por cromatografia em camada delgada}

As características fitoquímicas das tinturas foram avaliadas empregando-se um método modificado para cromatografia em camada delgada (CCD) previamente descrito (Wagner, Bladt, 1995). Para tal análise, foi empregada casca de Stryphnodendron barbatiman, gentilmente cedida pelo Grupo Centroflora, Botucatu - SP, da qual uma tintura-mãe padrão foi obtida a partir de $1 \mathrm{~g}$ da casca em $10 \mathrm{~mL}$ de solução hidroetanólica $(70 \% \mathrm{v} / \mathrm{v})$ por $10 \mathrm{minu}-$ tos a $70^{\circ} \mathrm{C}$ (Prista, Alves, Morgado, 1979), servindo como padrão de referência para detecção da presença e/ou quantidade de constituintes nos materiais avaliados. A identidade e autenticidade botânica desta planta foram confirmadas pelo farmacobotânico Professor Dr. Fernando de Oliveira do Centro de Ciências Biológicas e da Saúde, Universidade São Francisco, Bragança Paulista, São Paulo, Brasil.

Foram aplicados $5 \mu \mathrm{L}$ de cada tintura em cromatoplaca sílica gel 60F254 (Merck), utilizando-se uma mistura de acetato de etila: ácido fórmico: água (8:1:1, v/ $\mathrm{v} / \mathrm{v} /$ ) como fase móvel. A visualização foi feita através de 
nebulização de ácido sulfúrico 5 \% seguida da nebulização de vanilina $1 \%$ como agente cromogênico.

\section{RESULTADOS}

Na Tabela I, são apresentados os resultados obtidos da avaliação das características físico-químicas de tinturas de barbatimão. Os valores médios de $\mathrm{pH}$ e densidade, 4,87 e 0,93 , respectivamente, revelaram que não há diferença significativa entre as amostras uma vez que os desvios padrões relativos obtidos foram de 16,41 e 2,61 \%, respectivamente. Em relação aos valores do resíduo seco, variação significativa foi observada entre as amostras, considerando-se que o DPR foi de $66 \%$. Esta diferença se mostrou evidente principalmente entre as tinturas B e C. Resultado similar foi obtido na determinação de taninos.

A Figura 1 mostra o resultado da análise cromatográfica em cromatoplaca das amostras comparadas com a tintura-mãe padrão de barbatimão. Observa-se que a constituição fitoquímica das quatro tinturas avaliadas é semelhante, porém é evidente, através da intensidade da cor das manchas na cromatoplaca, a diferença nas concentrações dos componentes químicos das amostras. Assim, considerando os resultados obtidos nas análises físico-químicas e fitoquímica, a tintura $\mathrm{B}$ apresentou-se mais concentrada e o inverso foi observado para a amostra C, ou seja, a tintura mais diluída dentre as avaliadas neste estudo.

\section{DISCUSSÃO}

Em todo o mundo, encontra-se em expansão o comércio de plantas medicinais e produtos fitoterápicos em virtude de diversos fatores como o próprio modismo, alto custo e efeitos indesejáveis dos medicamentos sintéticos. Porém, a má qualidade e falta de padronização destes produtos, especialmente os comercializados nas farmácias brasileiras, é de conhecimento (Farias et al., 1985; Stelfelld, 1955) e podem comprometer a importância que estas plantas medicinais possam representar na saúde pública brasileira, confirmando a necessidade urgente da vigilância de fitoterápicos. Desta

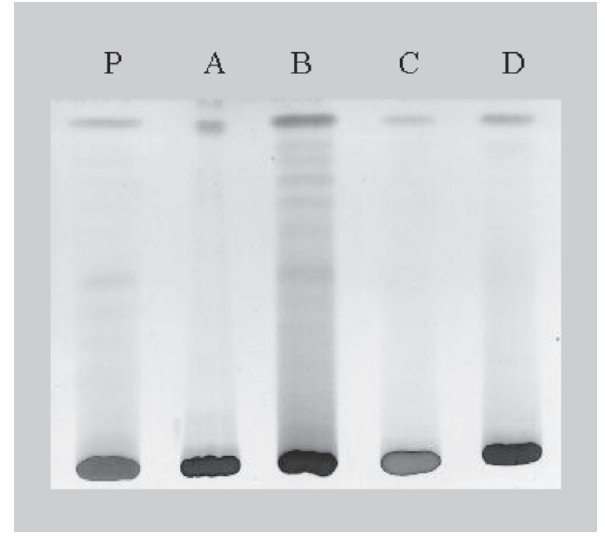

FIGURA 1 - Cromatografia em camada delgada, onde A, $\mathrm{B}, \mathrm{C}$ e $\mathrm{D}$ correspondem às amostras das tinturas avaliadas e P tintura-mãe padrão. Cromatoplaca 10 x $8 \mathrm{~cm}$ de sílica gel previamente ativada em estufa a $105^{\circ} \mathrm{C}$ por 20 minutos. $5 \mu \mathrm{L}$ de cada tintura e tintura-mãe padrão foram aplicados. Fase móvel: acetato de etila: ácido fórmico: água (8:1:1, v/v/v). Revelação: solução de ácido sulfúrico $5 \%$ em etanol seguida de vanilina $1 \%$ em etanol.

forma, foi elaborada uma portaria pelo Ministério da Saúde para regulamentar os procedimentos de produção destes medicamentos (MS, 1995).

Baseado nisto, este estudo avaliou as possíveis diferenças qualitativas e quantitativas de tinturas de barbatimão disponíveis no mercado, enfocando principalmente as características físico-químicas e fitoquímicas.

Os resultados obtidos da avaliação das características físico-químicas de tinturas de barbatimão revelaram diferenças significativas entre as amostras quando os parâmetros analisados foram o resíduo seco e teor de taninos. Já em relação à constituição fitoquímica, houve similaridade entre as tinturas avaliadas, porém diferença entre a concentração da composição química foi detectada através das manchas obtidas com a CCD, evidenciando que há diferença na qualidade das tinturas. A qualidade de um produto é dada por um conjunto de fatores que incluem desde a matéria-prima, controle do processamento, da forma farmacêutica, até a bula,

TABELA I - Comparação das características físico-químicas avaliadas nas tinturas de barbatimão de diferentes fabricantes.

\begin{tabular}{lccccccc}
\hline Parâmetros & \multicolumn{5}{c}{ Fornecedores } & & Métodos \\
& $\mathrm{A}$ & $\mathrm{B}$ & $\mathrm{C}$ & $\mathrm{D}$ & Média & DPR*(\%) & \\
\hline $\mathrm{pH}$ & 3,81 & 5,75 & 4,95 & 4,98 & 4,87 & 16,41 & Físico-químico \\
Resíduo seco $(\%)$ & 4,50 & 5,30 & 0,70 & 2,20 & 3,17 & 66,43 & Espectrofotométrico \\
Densidade $(\mathrm{g} / \mathrm{mL})$ & 0,96 & 0,90 & 0,93 & 0,92 & 0,93 & 2,61 & Físico \\
Teor de taninos $(\%)$ & 0,64 & 1,19 & 0,33 & 0,48 & 0,65 & 58,25 & Espectrofotométrico \\
\hline
\end{tabular}

* $\mathrm{DPR}=$ desvio padrão relativo 
a embalagem e a propaganda. Todos estes parâmetros podem afetar a segurança e eficácia do produto e causar prejuízos ao consumidor especialmente porque fitoterápicos, na sua grande maioria, são produtos cuja venda independe de prescrição médica. Com relação à qualidade dos insumos vegetais, os problemas mais freqüentes são o da adulteração, a não uniformidade da composição química e as contaminações como poeira, metais pesados, entre outros. A adulteração pode ser devido à substituição da parte usada do vegetal, contaminação com outras espécies vegetais ou total substituição do vegetal (Oliveira, 2000) devido principalmente à utilização da denominação popular, que varia de um lugar para outro, tanto na comercialização como na aquisição da matéria-prima. Assim, torna-se imprescindível que todo material adquirido pelos laboratórios seja analisado por profissionais capacitados, atestando a autenticidade e qualidade da matéria-prima. Em adição, composição química constante é garantia da uniformidade do produto final e desigualdades de um mesmo produto são frequentemente constatadas através da análise cromatográfica como conseqüência da adulteração e/ou irregularidade na época e local da coleta. Com os resultados obtidos neste estudo, percebe-se que não há adulteração em relação ao insumo vegetal quando comparado à solução obtida com a tintura-mãe padrão, ou seja, todas são provenientes de barbatimão. No entanto, diferenças nas concentrações dos componentes químicos foram evidenciadas, revelando que, embora exista no Brasil a Farmacopéia que descreve um método geral de preparação de tinturas, o que se verifica é que não há uma padronização no procedimento entre os fabricantes. Porém, como já anteriormente citado, existem outros fatores que devem ser considerados, tais como a idade da planta, local e época da colheita, qualidade do solvente empregado, e outros correlatos como a embalagem utilizada (hermética ou não) bem como a evaporação do produto durante a estocagem o que pode acarretar na concentração de seus componentes. Além disto, o conteúdo de taninos pode variar de forma significativa dependendo do método de extração empregado. Adeterminação de taninos em casca de 25 espécies de Eucalyptus revelou que o método de extração empregando solventes orgânicos (tolueno e etanol), para a maioria das espécies, promoveu uma maior extração de taninos comparado ao método da água quente (Trugilho et al., 2003). Com relação ao barbatimão, Jacobson e colaboradores (2005) avaliaram a influência de fatores edáficos na produção de fenóis totais e taninos de duas espécies (Stryphnodendron adstringens e S. polyphyllum), observando-se que solos com baixa fertilidade química e período chuvoso estão associados com maiores níveis de fenóis totais e taninos. Finalmente, conclui-se que, apesar da regulamentação da produção e comercialização de fitoterápicos, considerados medicamen- tos e sujeitos a estudos de eficácia, segurança e qualidade, existem variações qualitativas e quantitativas entre as amostras das tinturas de barbatimão avaliadas neste estudo, embora todas sejam provenientes de barbatimão.

\section{ABSTRACT}

\section{Evaluation of physico-chemical and phytochemical characteristics of different tinctures of barbatimão (Stryphnodendron barbatiman)}

As a consequence of the large distribution and use of medicinal plants, the industries are producing products based on plant species in various pharmaceutical forms, which have been commercialized in pharmacies and natural products homes. However, there is no guarantee for the vast majority of these products, as to their effectiveness, safety and quality, which may cause risks to the health of consumers. Therefore it is important the establishment of standardized protocols of quality control for phytotherapeutic products. Tinctures of barbatimão are available in the Brazilian market proceeding from diverse manufacturers. With the purpose to evaluate the difference between the quality of tinctures of barbatimão proceeding from four manufacturers, a comparative study of physico-chemical and phytochemical characteristics was carried out. For physico-chemical analysis, the $\mathrm{pH}$, density, dry residue and tannins content were evaluated. The phytochemical analysis was made using thin layer chromatography. The differences observed in physicochemical and phytochemical characteristics had evidenced the lack of standardization in the production of these tinctures.

UNITERMS: Barbatimão. Tinctures/physico-chemial analysis.

\section{REFERÊNCIAS BIBLIOGRÁFICAS}

BRASIL. Portaria n. 6, de 31 de Janeiro de 1995. Estabelece definições e procedimentos de registro de produtos fitoterápicos, e dá outras providências. Diário Oficial da União, Brasília, DF, 06 fev.1995.

BRASIL. Resolução RDC n.48, de 16 de março de 2004. Dispõe sobre o registro de medicamentos fitoterápicos. Diário Oficial da União, Brasília, DF, 18 mar. 2004.

CAÑIGUERAL, S.; DELLACASSA, E.; BANDONI, A.L. Plantas medicinales y Fitoterapia: indicadores de dependencia o factores de desarrollo? Acta Farm. Bonaer. v.22, p.265-278, 2003 . 
DE MELLO, J.P.; PETEREIT, F.; NAHRSEDT, A. Flavan3-ols and prodelphinidins from Stryphnodrendron adstrigens. Phytochemistry, v.441, p.807-813, 1996.

DESMICHELLE, G. O medicamento homeopático: fabricação, controle, legislação. In: MERCIER, L. (ORG.). Homeopatia princípios básicos. São Paulo: Organização Andrei, 1987. p.143-149.

DI STASI, L.C. Plantas medicinais: arte e ciência: um guia de estudo interdisciplinar. São Paulo: UNESP, 1996. 230p.

EUROPEAN Pharmacopoeia. Directorate for the quality of medicines of the Council of Europe (EDQM). 5.ed. Strasbourg: Council of Europe, 2005. 3000p.

FARIAS, M.R.; SCHENKEL, E.P.; BERGOLD, A.M.; PETROVICK, P.R. O problema da qualidade dos fitoterápicos. Cad. Farmácia, v.1, p.73-82, 1985.

FARMACOPÉIA Brasileira. 4.ed. São Paulo: Atheneu, 1988. pt.1,300p.

FELFILI, J.M.; DA SILVA JÚNIOR, M.C.; DIAS, B.J.; REZENDE, A.V. Estudo fenológico de Stryphnodendron adstringens (Mart.) Coville no cerrado sensu stricto da Fazenda Água Limpa no Distrito Federal, Brasil. Rev. Bras. Bot., v.22, p.83-90, 1999.

GUARIM NETO, G. Plantas utilizadas na medicina popular do Estado de Mato Grosso. Brasília: CNPq/UFMT, 1987. 58p.

JACOBSON, T.K.B.; GARCIA, J.; SANTOS, S.C.; DUARTE, J.B.; FARIAS, J.G.; KLIEMANN, H.J. Influência de fatores edáficos na produção de fenóis totais e taninos de duas espécies de barbatimão (Stryphnodendron sp.). Pesqui. Agropecu. Trop., v.35, p.163-169, 2005.

LOPES, G.C.; SANCHES, A.C.C.; NAKAMURA, C.V.; DIAS-FILHO, B.P.; HERNANDES, L.; DE MELLO, J.C.P. Influence of extracts of Stryphnodendron polyphyllum Mart. And Stryphnodendron obovatum Benth, on the cicatrisation of cutaneous wounds in rats. $J$. Ethnopharmacol., v.99, p.265-272, 2005.

LORENZI, H. Árvores brasileiras. Nova Odessa: Plantarum, 1992. 373p.
MAGALHÃES, P.C.; RODRIGUES, W.A.; DURÃES, F.M. Tanino no grão de sorgo: bases fisiológicas e métodos de determinação. Sete Lagoas: EMBRAPA-CNPMS, 1997. $26 \mathrm{p}$.

MONTEIRO, J.M.; ALBUQUERQUE, U.P.; LINS-NETO, E.M.F.; ARAÚJO, E.L.; ALBUQUERQUE, M.M.; AMORIM, E.L.C. The effects of seasonal climate changes in the caatinga on tannin levels in Myracrodruon urundeuva (Engl.) Fr. All. and Anadenanthera colubrine (Vell.) Brenan. Rev. Bras. Farmacogn., v.16, p.338-344, 2006.

NEWALL, C.A.; ANDERSON, L.A.; PHILLIPSON, J.D. Plantas medicinais: guia para profissional de saúde. São Paulo: Premier, 1996. 296p.

OLIVEIRA, F.; AKISUE, G. Fundamentos da farmacobotânica. 2.ed. São Paulo: Atheneu, 2000. 178p.

PANIZZA, S.; ROCHA, A.B.; GECCHI, R.; SOUZA E SILVA, R.A.P. Stryphnodendron adstrigens barbadetiman (Vell.) Martius: teor de tanino na casca e sua propriedade cicatrizante. Rev. Bras. Ciênc. Farm., v.10, p.101-106, 1988.

POZETTI, G.L.; CABRERA, A.; BERNARDI, A.C. Controle cromatográfico da qualidade de algumas tinturas-mães de Calendula officinalis. Pesqui. homeopát., v.2, p.33-40, 1986.

PRISTA, L.N.; ALVES, A.C.; MORGADO, R. Formas farmacêuticas obtidas por dispersão molecular. In: Técnica farmacêutica e farmácia galênica. 2.ed. Lisboa: Fundação Calouste Gulbenkian, 1979. p.14511469.

RIZZINI, C.T.; MORS, W.B. Botânica econômica brasileira. São Paulo: EDUSP, 1976. 227p.

SANTOS, S.C.; COSTA, W.F.; RIBEIRO, J.P.; GUIMARÃES, D.O.; FERRI, P.H.; FERREIRA, H.D.; SERAPHIN, J.C. Tannin composition of barbatimão species. Fitoterapia, v.73, p.292-299, 2002.

SINGLENTON, V.L.; ORTHOFER, R.; LAMUELARAVENTÓS, R.M. Analysis of total phenols and other oxidation substrates and antioxidants by means of FolinCiocalteau reagent. Meth. Enzymol., v.299, p.152-178, 1999. 
STELLFELD, C. Da necessidade da regulamentação do comércio de plantas medicinais. Trib. Farm., v.22, p.185189, 1955.

TEIXEIRA, M.L.; SOARES, A.R.; SCOLFORO, J.R.S. Variação do teor de tanino da casca de barbatimão (Stryphnodendron adstringens Mart. Coville) em 10 locais de Minas Gerais. Cienc. Prat., v.14, p.229-232, 1990.

TRUGILHO, P.F.; MORI, F.A.; LIMA, J.T.; CARDOSO, D.P. Determinação do teor de taninos na casca de Eucalyptus spp. Rev. Cerne, v.9, p.246-254, 2003.
VIEIRA, R.A. Validação científica de plantas medicinais como fator catalisador no desenvolvimento da indústria farmacêutica nacional. Meio Ambient. Saúde, v.2, p.5764, 2001.

WAGNER, H.; BLADT, S. Polyphenols. In: Plant drug analysis: a thin layer chromatography atlas. 2 .ed. Heidelberg: Springer, 1995. p.22-24.

Recebido para publicação em 06 de agosto de 2007 Aceito para publicação em 09 de janeiro de 2008 